\title{
A Planar Multi-Antenna Design Supporting 3.4-4.2 GHz for 5G Smartphone Applications
}

\author{
Naser Ojaroudi Parchin ${ }^{* 1}$, Haleh Jahanbakhsh Basherlou ${ }^{2}$, Yasir I. A. Al-Yasir ${ }^{3}$, Yim F. Hu ${ }^{4}$, \\ and Raed A. Abd-Alhameed ${ }^{5,6}$ \\ $\left\{\right.$ N.OjaroudiParchin@Bradford.ac.uk $\left.{ }^{1 *}\right\}$ \\ Faculty of Engineering and Informatics, University of Bradford, Bradford BD7 1DP, UK $\mathrm{UK}^{1,3,4,5}$ \\ Bradford College, Bradford, West Yorkshire, BD7 1AY, UK² \\ Information and Communication Engineering Department, Basrah University College of \\ Science and Technology, $\operatorname{Iraq}^{6}$
}

\begin{abstract}
A MIMO antenna array with suitable features for 5G mobile phone applications is presented in this study. With $75 \times 150 \times 0.8 \mathrm{~mm}^{3} \mathrm{FR}-4$ dielectric, two rows of tiny monopole antenna arrays are mounted on the right and left sides of the mainboard. A modified T-shaped resonator with discontinuous feeding and a protruded L-shaped strip make up the monopole element's schematic. The design parts have all been etched into the same layer of the ground. The proposed array is extremely compact and takes up very little space on the PCB plane. The presented MIMO smartphone antenna is designed to support the sub $6 \mathrm{GHz} 5 \mathrm{G}$ network and provides a 3.4-4.2 GHz operation band. Also, due to the arrangement of the elements, it could operate as two sets of $1 \times 4$ phased arrays with high-gain and beam steerable radiation patterns. The MIMO performance, user-hand effect, and critical properties of the introduced monopole antenna array design are studied.
\end{abstract}

Keywords: 5G, MIMO system, mobile-phone antenna, a monopole antenna, wireless communications.

\section{Introduction}

Multiple-input-multiple-output (MIMO) with multiple radiation elements has been gaining a lot of interest for next-generation $(5 \mathrm{G})$ communication networks to meet the rising demand for transmission rate and throughput for various applications [1-5]. The 5G communication system also requires an array antenna with an enhanced number of antenna elements and dualpolarization capability in the case of massive MIMO [6-10]. MIMO antennas have been seen in a variety of smart and portable devices, including smartphones, laptops, and tablets. Many problems must be overcome in order to achieve an efficient MIMO operation for user equipment [11-18].

Monopole resonators, among the numerous forms of MIMO antennas, are more ideal due to their appealing properties. Recently, various MIMO antennas with narrow bandwidth and large sizes are represented for sub $6 \mathrm{GHz}$ mobile-phone applications [19-25]. We introduce here a MIMO monopole antenna array with eight elements and a wide operation band of 3.44.2 GHz. The employed monopole antenna elements have low profiles, and their configuration 
contains a modified T-shaped resonator with discrete feeding and a protruded L-shaped strip. The experiment was conducted using the EM simulation CST programme [26].

The scattering parameters, patterns, efficiency, and gain of the planned array have all been evaluated and achieved for best performance. Furthermore, the suggested 5G antenna performs well in the proximity of the user-hand phantom. Based on the research work discussed in the following, sufficient characteristics are achieved with straightforward structure and novel. Section 2 presents the schematic and design details. Its fundamental characteristics and the MIMO antenna performance are illustrated in section 3. Section 4 investigates the antenna radiation behaviour with a double-hand phantom. The last section of the paper will conclude this research study.

\section{Configuration and Geometrical Details}

The introduced phased array is depicted schematically in Fig 1. It includes eight monopole antennas that are arrayed in two sets of 14 linear array on both sides of the PCB plane, as seen. The antenna was mounted on the same layer of the ground. Table 1 lists the antenna parameters and their values.

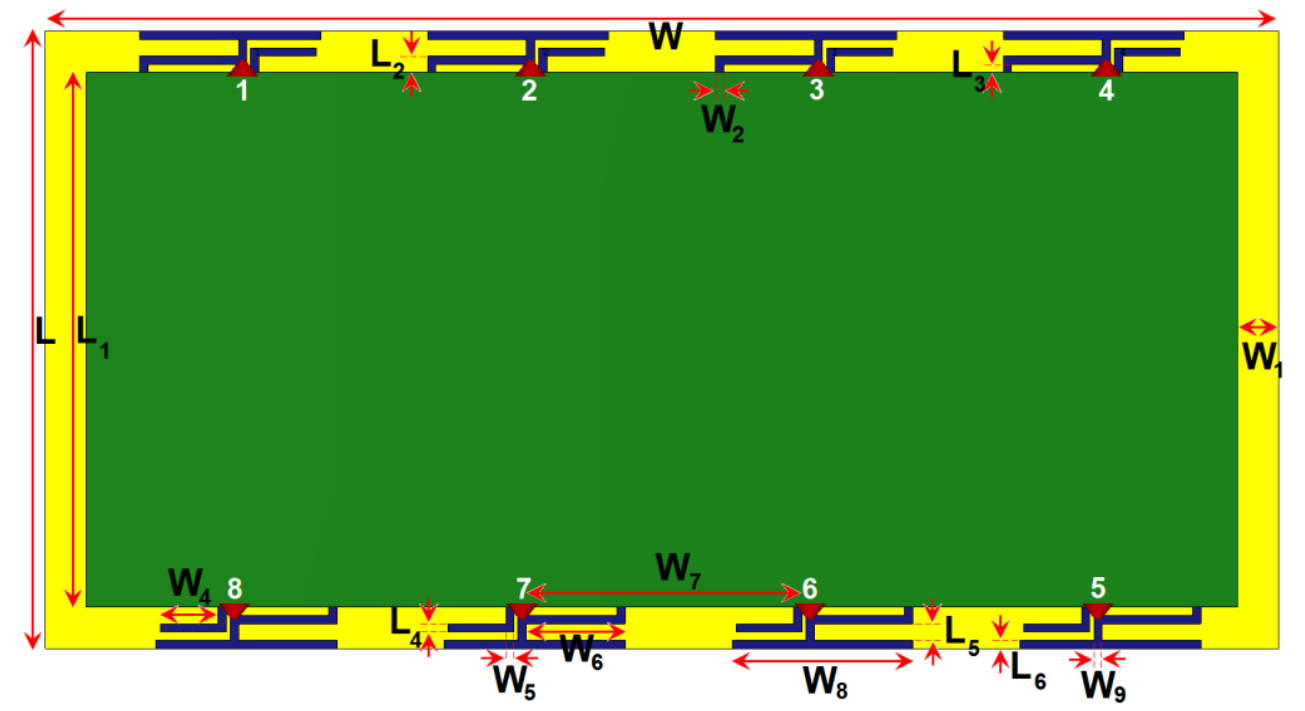

Fig 1. Antenna elements handset.

Table 1. Dimension of the antenna structure all in $\mathrm{mm}$

\begin{tabular}{cccccccc}
\hline $\mathbf{W}$ & $\mathbf{L}$ & $\mathbf{W 1}$ & $\mathbf{L 1}$ & $\mathbf{W}_{\mathbf{2}}$ & $\mathbf{L 2}$ & $\mathbf{W}_{\mathbf{3}}$ & $\mathbf{L}_{3}$ \\
\hline 150 & 75 & 5 & 65 & 0.5 & 2 & 0.5 & 1 \\
\hline $\mathrm{W} 4$ & L4 & W5 & L5 & W6 & L6 & W7 & W8 \\
\hline 7 & 1 & 0.5 & 2 & 12 & 1 & 35 & 22 \\
\hline
\end{tabular}




\section{Characteristics of the MIMO Array}

Figs 2 (a) and (b) illustrate $\mathrm{S}_{\mathrm{nn}}$ (scattering parameters) and $\mathrm{S}_{\mathrm{mn}}$ (transmission coefficient) properties of the designed MIMO monopole array, respectively. As seen from Fig 2 (a), a wide operating frequency band of $800 \mathrm{MHz}$ for $S_{n n} \leq-10$ and $1400 \mathrm{MHz}$ for $S_{n n}$ $\leq-6 \mathrm{~dB}$ is obtained. In addition, sufficient mutual couplings have been obtained as illustrated in Fig 2 (b).

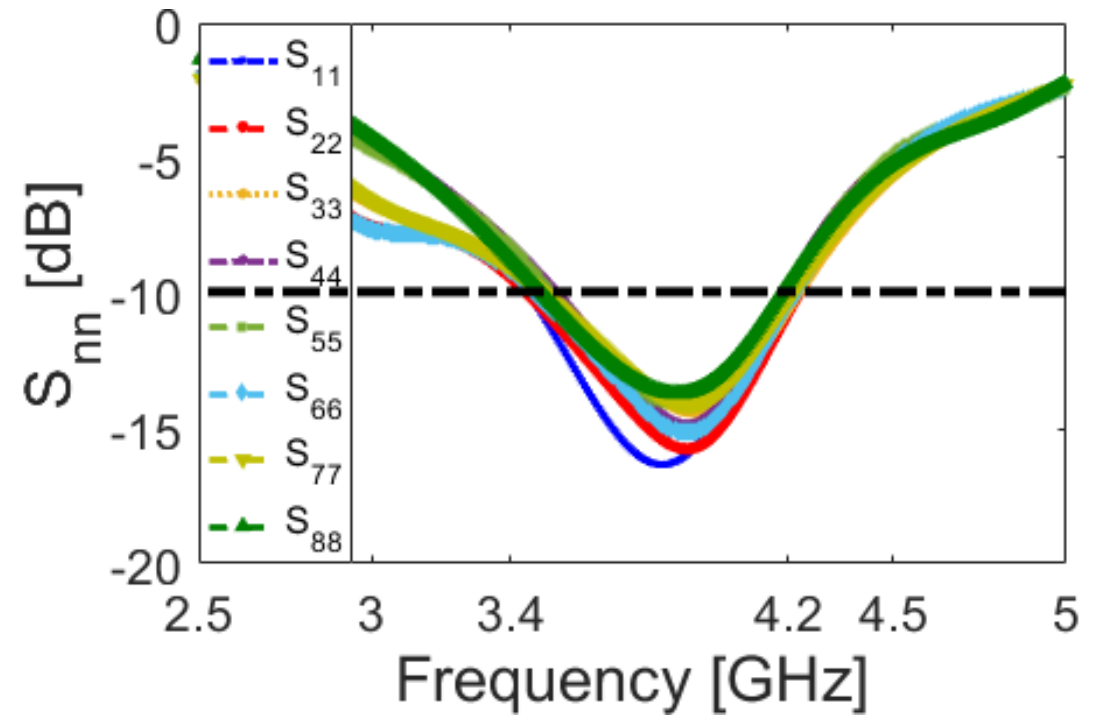

(a)

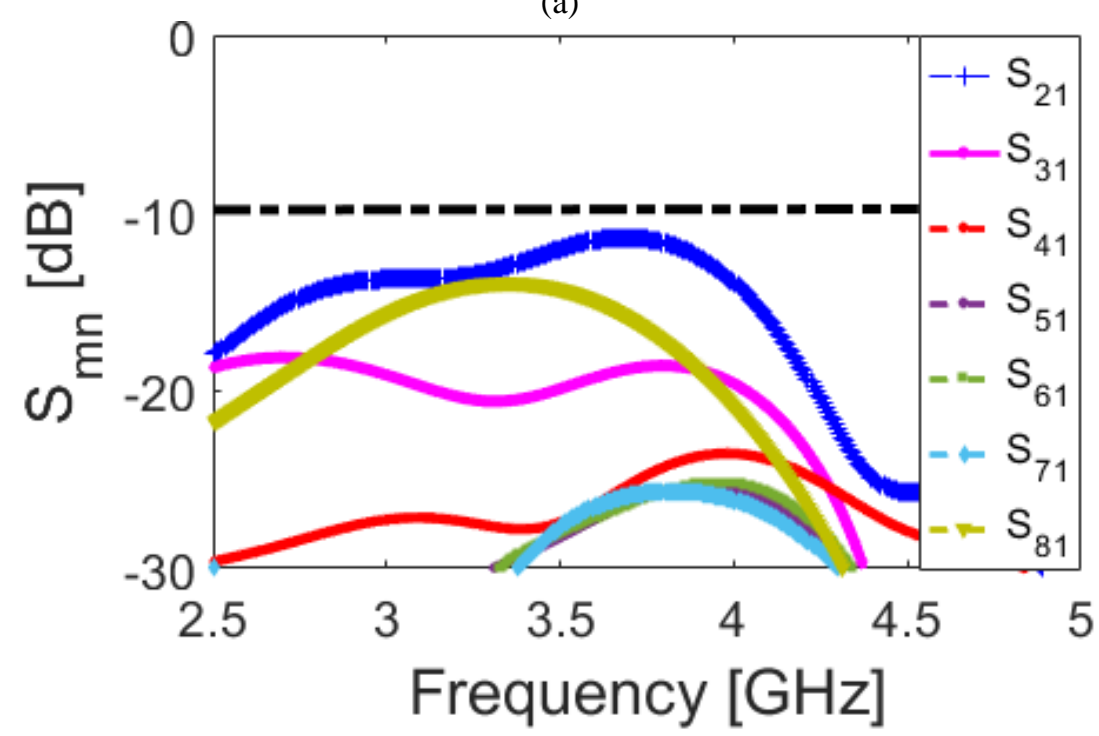

(b)

Fig 2. Scattering parameters, (a) $S_{n n}$ and (b) $S_{m n}$. 
Figures 3 (a) and 3 (b) illustrate the efficiencies of the proposed antenna elements. At 3.44.2 $\mathrm{GHz}$, it has been observed that sufficient and high-efficiency results have been produced. A higher than $80 \%$ radiation efficiency characteristic is attained for $3.44 \mathrm{GHz}$, as illustrated in Fig 3 (a). Furthermore, as shown in Figure 3 (b), more than 65 percent of total efficiencies have been achieved [27-30].

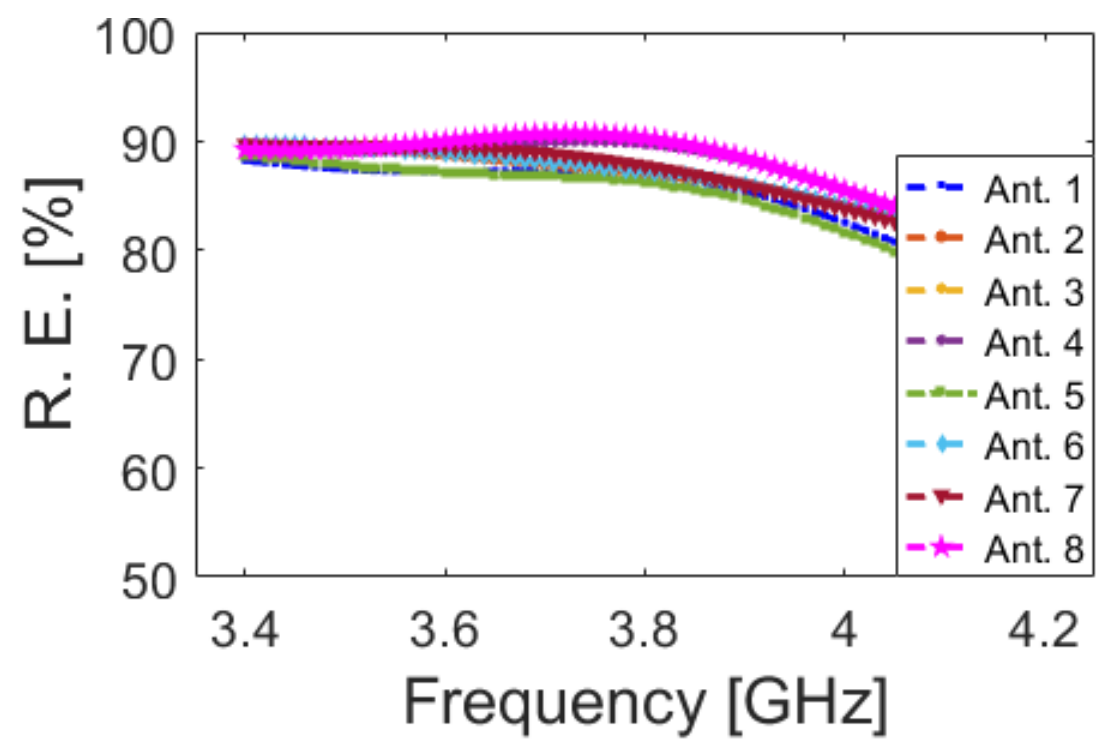

(a)

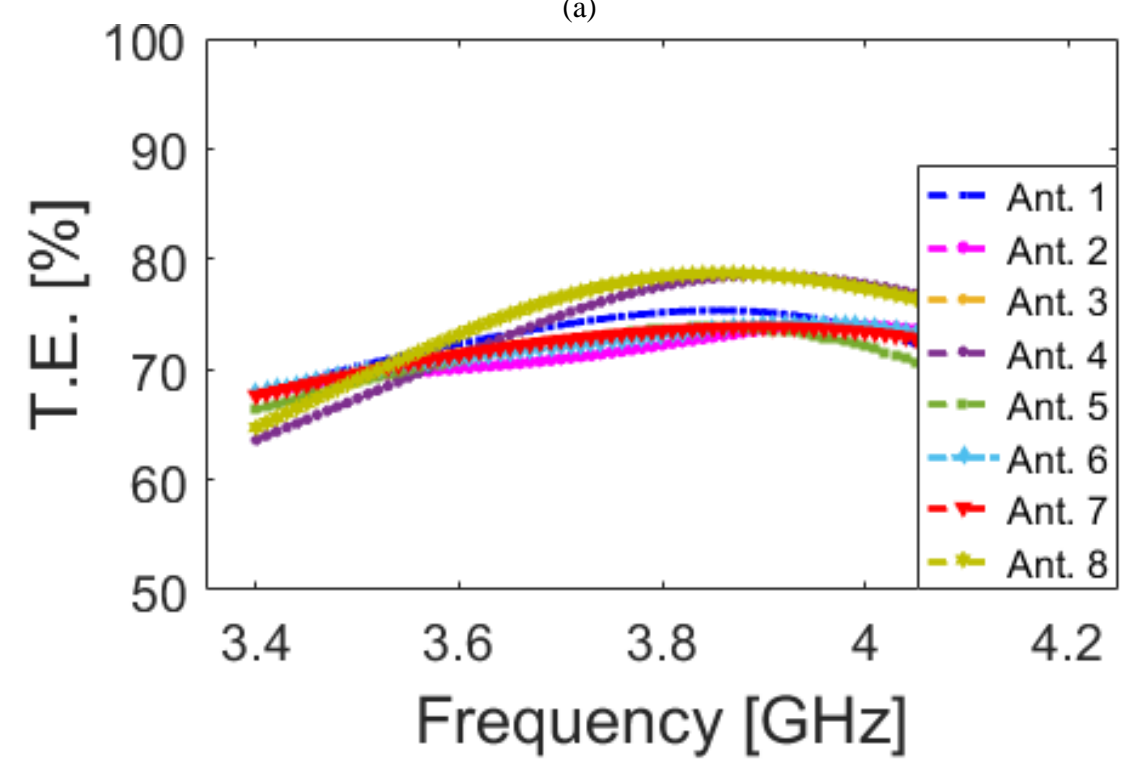

(b)

Fig 3. (a) Radiation efficiency and (b) total efficiency results. 


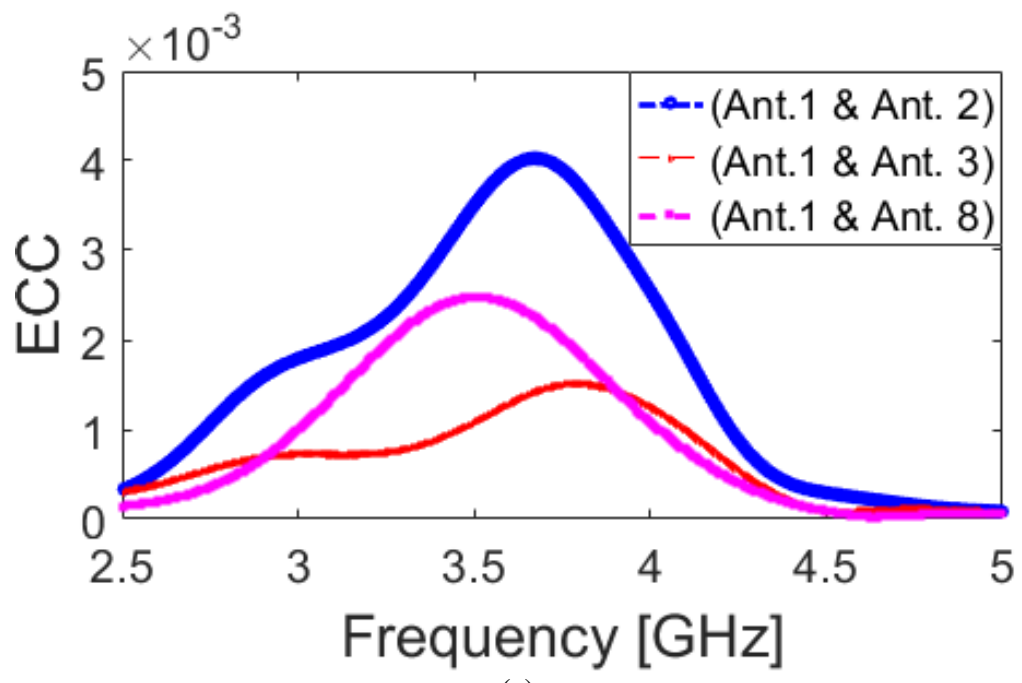

(a)

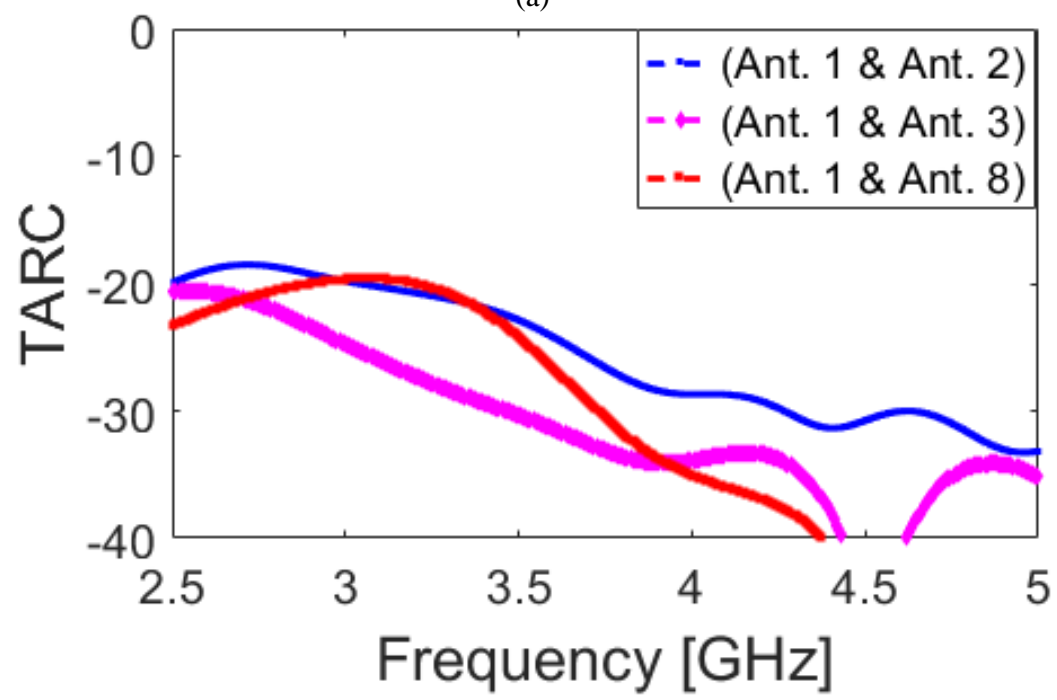

(b)

Fig 4. a) ECC, b) TARC properties.

ECC and TARC requirements are critical in MIMO systems and should be kept as small as possible [31-32]. The variations of these parameters for a closely spaced element are shown in Figures 4 (a) and (b). Within the operation band, these values are quite low. At the intermediate frequency $(3.8 \mathrm{GHz})$, the $3 \mathrm{D}$ fluctuation of the radiated field for the antenna elements is investigated and depicted in Fig. 5. This diagram illustrates the field shape and how the elements of the MIMO antenna may support different sides of the handset while also improving the design's performance [33-36]. In addition, good gain values are achieved for each antenna resonator. As mentioned earlier, the set of antennas could operate as two sets of $1 \times 4$ phased arrays with high-gain and beam-steerable radiation patterns. Fig 6 investigates the radiation performance of the sub-arrays at the resonance frequency $(3.8 \mathrm{GHz})$ and different 
scanning angles. According to obtained results, it is found that each set of $1 \times 4$ sub-array could provide high gain radiation beams with a wide beam-steering. Figure 7 plots and compares the main characteristics of the element and the main sub-array antenna over 3.4 to $4.2 \mathrm{GHz}$. As see, the element can provide $2-4.5 \mathrm{dBi}$ maximum gain while the sub-array exhibits a high maximum gain characteristic $(6-8.5 \mathrm{dBi})$ over the antenna operation band [37-42].

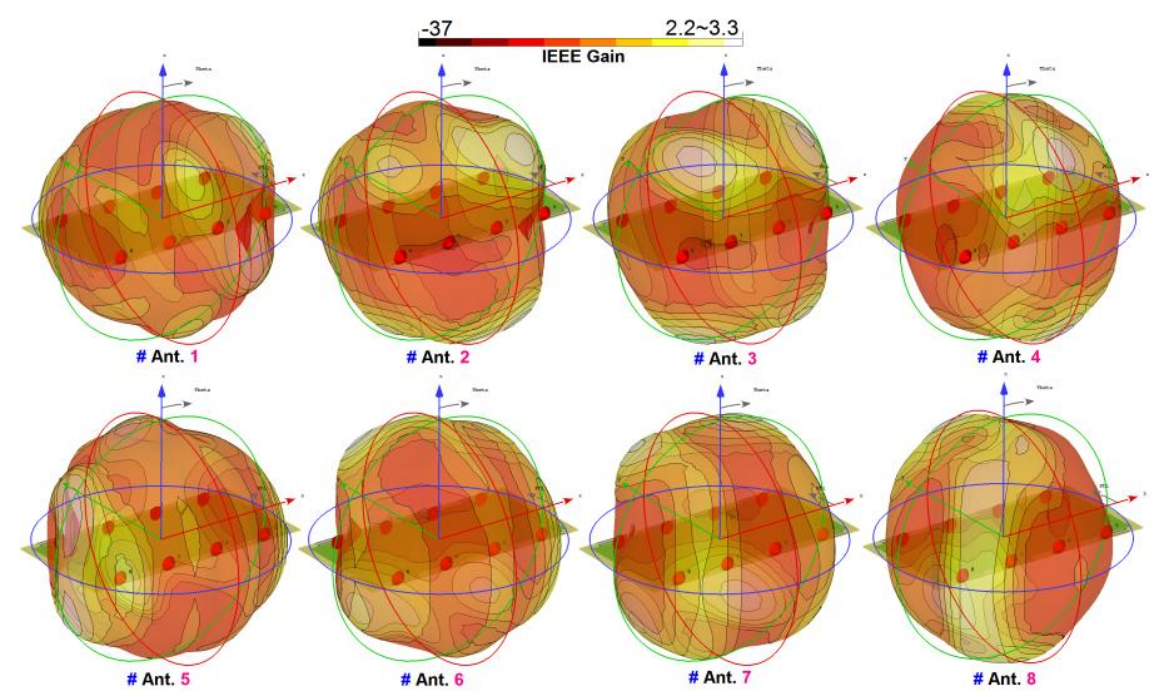

Fig. 5. 3D Radiations of each antenna element at $3.8 \mathrm{GHz}$.
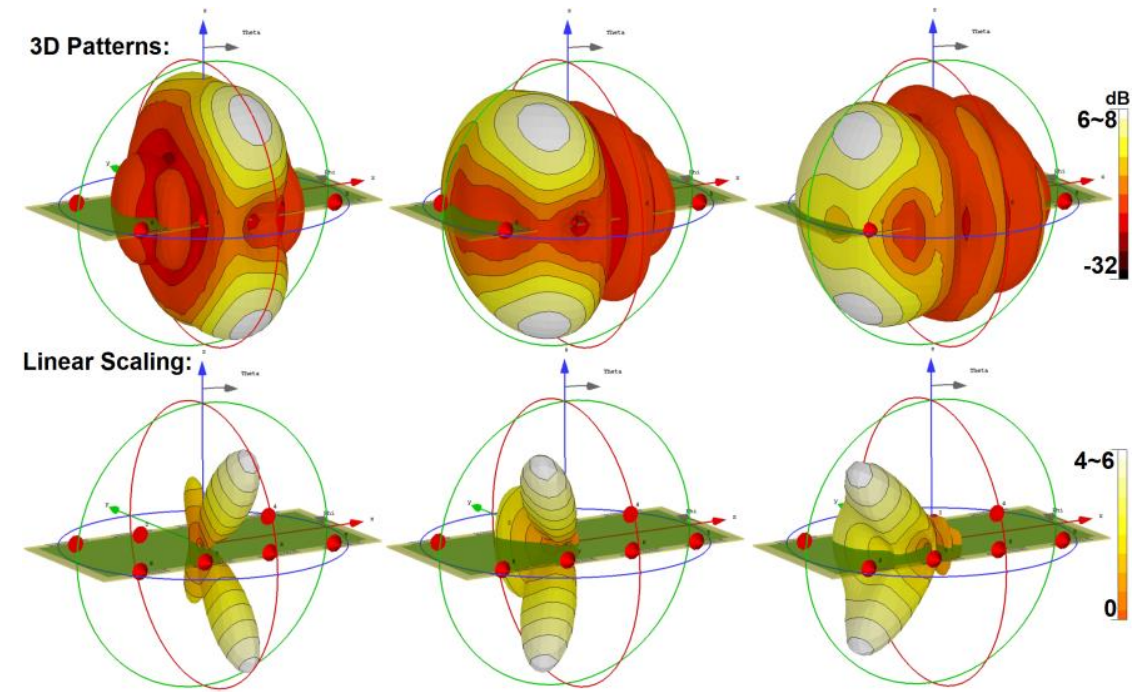

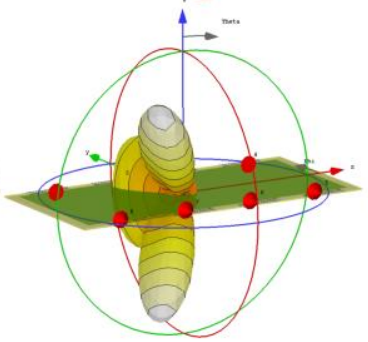

(b)

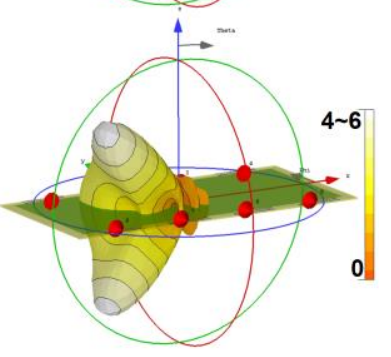

(c)

Fig 6. Beam patterns of the sub-arrays ( $1 \times 4$ linear array) at (a) 0 , (b) 30 , and (c) 60 degrees. 


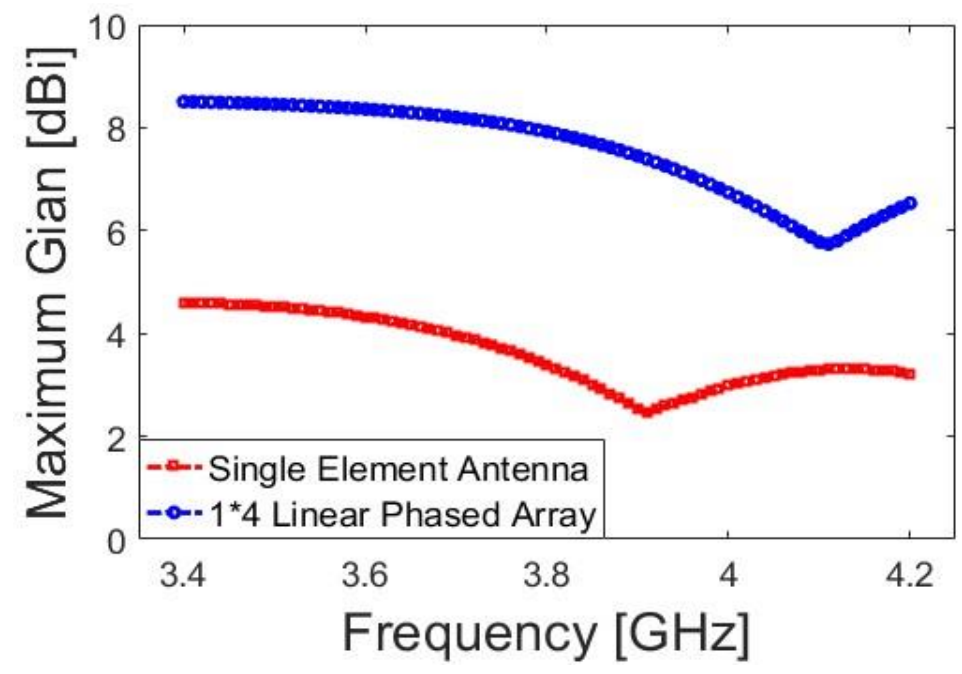

Fig 7. Max gains of a single element and the sub-array antennas.

\section{User-Hands Impact}

It is possible to lower the antenna performance with the user hand. This is also dependent on the antenna component's distance and positioning [43-45]. The features of the inserted antenna elements in the presence of the double-hands model are illustrated in the following section.. The placement and $S_{n n}$ results in the double-hand scenario are depicted in Figs. 8 and 9, respectively. As depicted, the introduced mobile antenna design exhibits sufficient $S_{n n}$ results supporting the target frequency band.

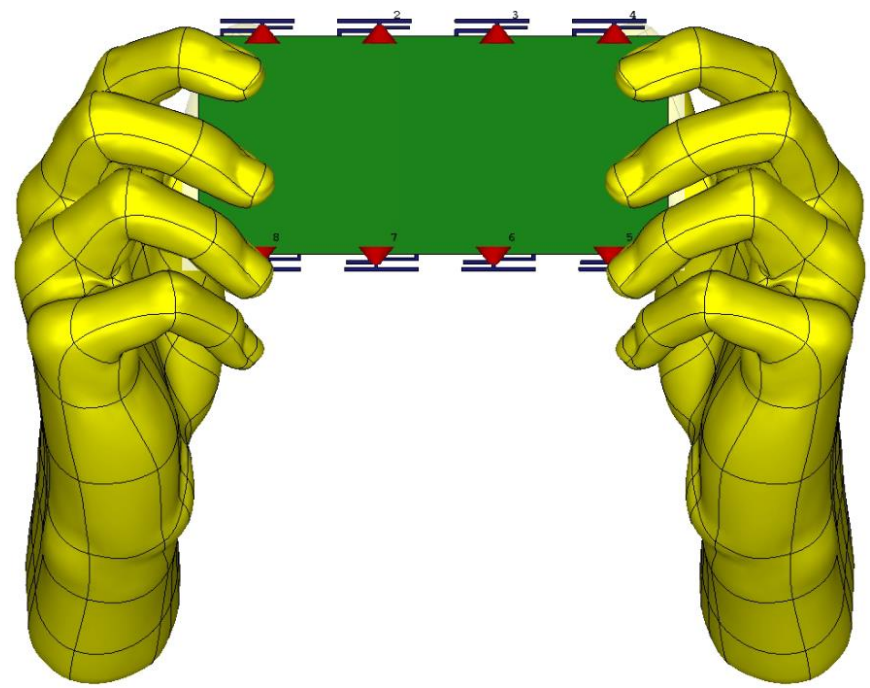

Fig 8. In double-hand mode, the antenna is positioned. 


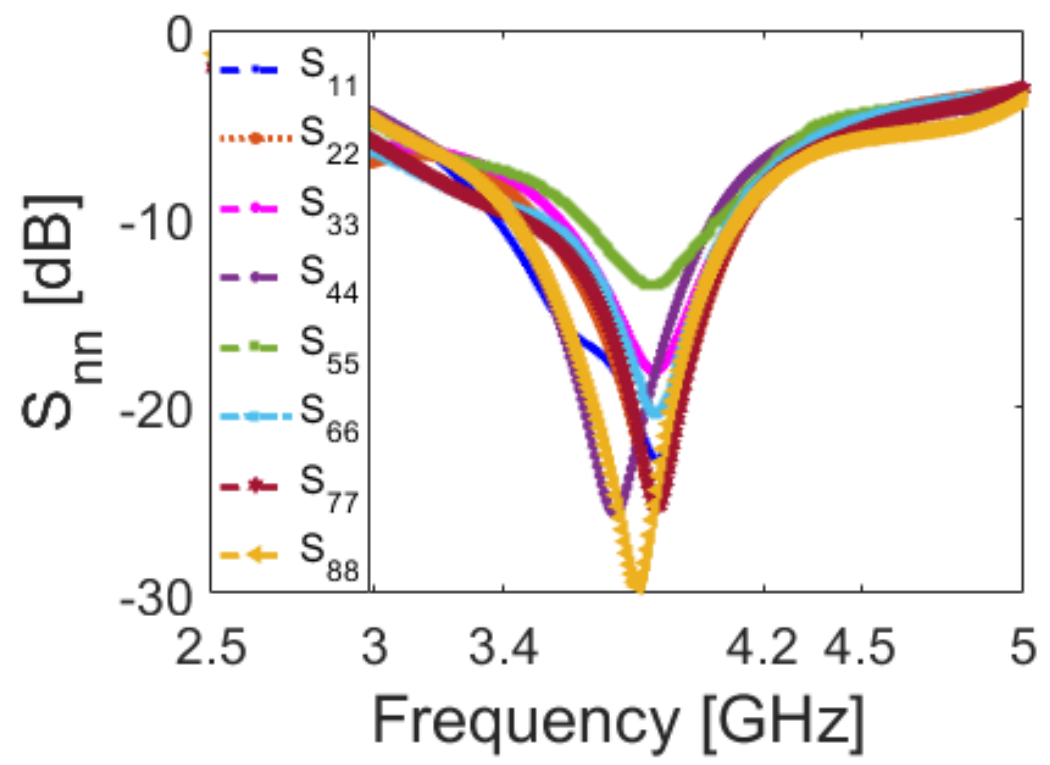

Fig 9. The variations of $S_{n n}$ with user's hand.

Furthermore, the suggested mobile-phone antenna has high efficiency (30 percent -60 percent). The antennas' efficiency levels are reduced, but not dramatically, as seen in Fig. 10. This could be owing to the used element's tiny and compact sizes, which are not covered by the user's hand. It's worth mentioning that the resonators partially encompassed by the phantom show the greatest decreases in performance [46].

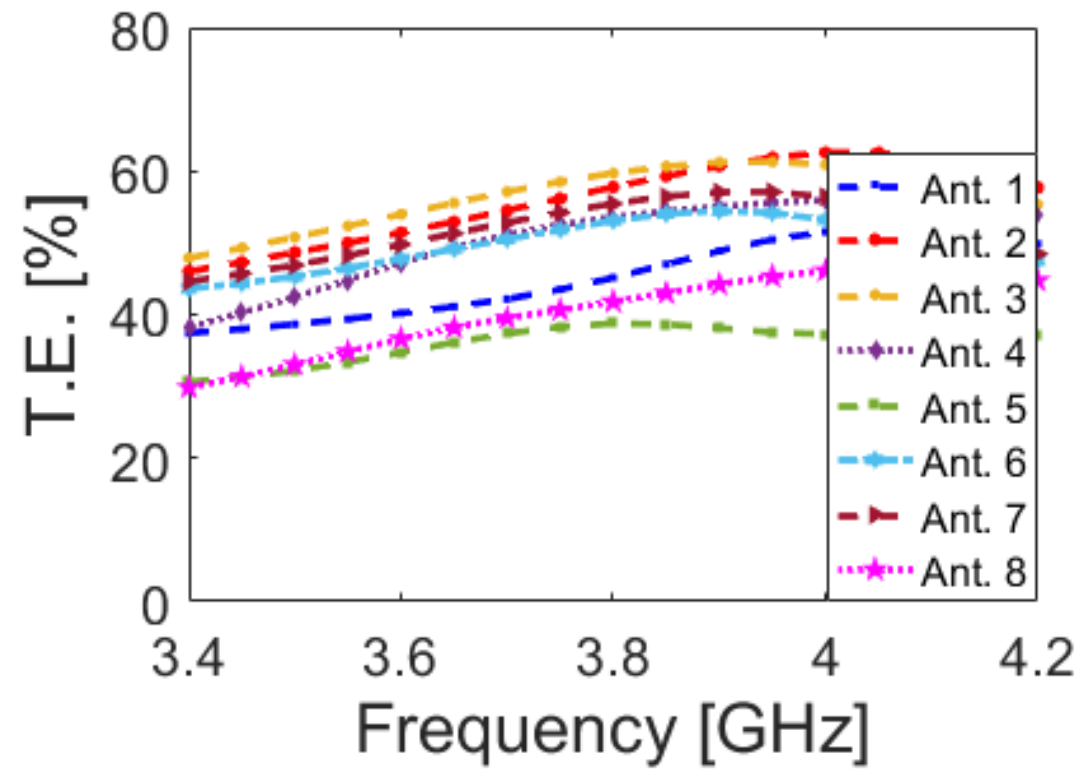

Fig. 10. Antenna total efficiencies with user's hand. 


\section{Conclusion}

For 5G mobile phone applications, an antenna array with compact resonators and large impedance bandwidth has been introduced. At both sides of mobile phone mainboard, eight small monopole antenna arrays are designed to function in the $3.4-4.2 \mathrm{GHz}$ sub- $6 \mathrm{GHz} 5 \mathrm{G}$ band. A modified T-shaped resonator with discrete feeding and a protruded L-shaped strip are included in each monopole antenna element. It also takes up a minimal amount of space on the PCB plane and has sufficient properties. The most important parameters of the designed handset were explored.

\section{Acknowledgment}

The European Union's Horizon 2020 innovation programme under grant agreement H2020MSCA-ITN-2016 SECRET-722424 and the UK Engineering and Physical Sciences Research Council (EPSRC) under grant EP/E022936/1 supported this research.

\section{References}

[1] Osseiran A, et al. Scenarios for 5G mobile and wireless communications: the vision of the METIS project. IEEE Commun. Mag. 2014, 52:26-35.

[2] Parchin, N. O. et al.: Microwave/RF components for 5G front-end systems. Avid Science. 2019, 1-200.

[3] Parchin, N. O. et al.: Wide-scan phased array antenna fed by coax-to-microstriplines for $5 \mathrm{G}$ cell phones. 2016 21st International Conference on Microwave, Radar and Wireless Communications. MIKON 2019, Krakow, Poland.

[4] Al-Yasir, Y. et al.: New radiation pattern-reconfigurable $60-\mathrm{GHz}$ antenna for 5G communications. Modern Printed Circuit Antennas, IntechOpen. 2019.

[5] Ullah, A. et al.: Coplanar waveguide antenna with defected ground structure for 5G millimeter wave communications. IEEE MENACOMM'19, 2019, Bahrain.

[6] Yang HH, Quel YQS. Massive MIMO meet small cell. SpringerBriefs in Electrical and Computer Engineering. 2017. DOI 10.1007/978-3-319-43715-6_2.

[7] Parchin, N. O. et al.: Dual-polarized MIMO antenna array design using miniaturized selfcomplementary structures for 5G smartphone applications. EuCAP 2019, Krakow, Poland.

[8] Mazloum, J. et al.: Compact triple-band S-shaped monopole diversity antenna for MIMO applications. ACES Journal, 2015, 28:975-980.

[9] Parchin, N. O. and Abd-Alhameed: A compact Vivaldi antenna array for 5G channel sounding applications. EuCAP 2018, London, UK.

[10] Parchin, N. O. et al.: Mobile-phone antenna array with diamond-ring slot elements for 5G massive MIMO systems. Electronics. 2019, 8:521.

[11] Zhang Z, Antenna Design for Mobile Devices. Wiley-IEEE Press. 2011, NJ, USA.

[12] Ojaroudi, N. and Ghadimi, N.: Dual-band CPW-fed slot antenna for LTE and WiBro applications. Microw. Opt. Technol. Lett. 2014, 56:1013-1015.

[13] Musavand, J. et al.: A Compact UWB Slot Antenna with Reconfigurable Band-Notched Function for Multimode Applications. ACES Journal, 2016, 31:14-18.

[14] Ojaroudiparchin, N. et al.: $8 \times 8$ planar phased array antenna with high efficiency and insensitivity properties for 5G mobile base stations. EuCAP 2016, Switzerland.

[15] Parchin, N. O. et al.: Frequency reconfigurable antenna array with compact end-fire radiators for 4G/5G mobile handsets. IEEE 2nd 5G World Forum. 5GWF 2019, Dresden, Germany.

[16] Ojaroudiparchin, N. et al.: Low-cost planar mm-Wave phased array antenna for use in mobile satellite (MSAT) platforms. Telecommunications Forum, TELFOR 2015, Belgrade, Serbia. 
[17] Ojaroudi, N.: A novel design of microstrip antenna with reconfigurable band rejection for cognitive radio applications. Microw. Opt. Technol. Lett. 2014, 56:2998-3003.

[18] Jensen M, Wallace J. A review of antennas and propagation for MIMO wireless communications. IEEE Trans. Antennas Propag. 2004, 52: 2810-2824.

[19] Abdullah M, et al. Eight-element antenna array at 3.5 GHz for MIMO wireless application. PIER C. 2017, 78: 209-217.

[20] Parchin NO, et al. Dual-polarized MIMO antenna array design using miniaturized selfcomplementary structures for 5G smartphone applications. EuCAP 2019, Krakow, Poland.

[21] $\mathrm{Li} \mathrm{Y}$, et al. High-isolation 3.5-GHz 8-antenna MIMO array using balanced open slot antenna element for 5G smartphones. IEEE Trans. Antennas Propag. 2019, 67: 3820-3830.

[22] Wang L, et al. Compact UWB MIMO antenna With high isolation using fence-type decoupling structure. IEEE Antennas and Wireless Propagation Letters. 2019, 8:1641-1645,

[23] Basherlou, H. J. et al.: MIMO monopole antenna design with improved isolation for 5G WiFi applications. International Journal of Electrical and Electronic Science. 2019, 7:1-5.

[24] Parchin, N. O. et al.: Multi-mode smartphone antenna array for 5G massive MIMO applications. EuCAP 2020, Copenhagen, Denmark.

[25] Chen WS, Lai YC, and Sim CYD, MIMO dongle antenna design for next generation mobile communication system. ICCEM. 2017, 320-322.

[26] CST Microwave Studio, ver. 2017, CST. 2017, Framingham, MA, USA.

[27] Parchin, N. O. et al.: Beam-steerable microstrip-fed bow-tie antenna array for fifth generation cellular communications. EuCAP 2016, Switzerland.

[28] Ullah. A, et al:. A Modified Design of Phased Array Antenna for UWB-5G Cellular Communications. 2020 IMDC-SDSP 8-10 April 2020, Antalya- Turkey

[29] Parchin, N. O. et al.: Dual-Polarized Array Antenna with Quasi-End-Fire Radiation for $28 \mathrm{GHz}$ 5G Mobile terminals. 2020 IMDC-SDSP 8-10 April Antalya- Turkey

[30] Al-Yasir, Y. et al.: New pattern reconfigurable circular disk antenna using two PIN diodes for WiMax/WiFi (IEEE 802.11a) applications. IEEE SMACD 2019, Lausanne, Switzerland.

[31] Sharawi MS, Advancements in MIMO antenna systems. Developments in antenna analysis and synthesis. 2018, Chapter-4. IET.

[32] Musavand A, et al. A compact UWB slot antenna with reconfigurable band-notched function for multimode applications. Appl Comp Electromagn Soc J. 2016, 31:14-18.

[33] Sharawi MS, Printed multi-band MIMO antenna systems and their performance metrics [wireless corner]. IEEE Antennas Propag. Mag. 2013, 55: 218-232.

[34] Parchin NO, Low-profile air-filled antenna for next generation wireless systems. Wirelss Personal Communications. 2017, 97: 3293-3300.

[35] Chen Q, et al. Design considerations for millimeter wave antennas within a chip package. IEEE International Workshop on Anticounterfeiting, Security, Identification. 2007, Xiamen, China.

[36] Abdollahi, M. M. et al.: Octave-band monopole antenna with a horseshoe ground plane. ACES Journal, 2015, 30:773-778.

[37] Zolghadr, J. et al.: UWB slot antenna with band-notched property with time domain modeling based on genetic algorithm optimization. ACES Journal, 2016, 31:926-932.

[38] Syrytsin I, et al.: Performance investigation of a mobile terminal phased array with user effects at $3.5 \mathrm{GHz}$ for LTE advanced. IEEE Antennas and Wireless Propagation Letters. 2017, 18471850 .

[39] Kamalvand, A. et al.: Omni-directional/multi-resonance CPW-fed small slot antenna for UWB applications. ACES Journal. 2013, 28:829-835.

[40] Ojaroudi, N.: Microstrip monopole antenna with dual band-stop function for ultrawideband applications. Microw. Opt. Technol. Lett. 2014, 56: 818-822.

[41] Valizade, A. et al.: Band-notch slot antenna with enhanced bandwidth by using $\Omega$-shaped strips protruded inside rectangular slots for UWB applications. ACES Journal, 2012, 27: 816-822. 
[42] Siahkal-Mahalle, B. H. et al.: A new design of small square monopole antenna with enhanced bandwidth by using cross-shaped slot and conductor-backed plane. Microwave Opt Technol Lett. 2012, 54:2656-2659.

[43] Khan R, et al. User influence on mobile terminal antennas: A review of challenges and potential solution for 5G antennas. IEEE Access. 2018, 6:77695-77715.

[44] Yasir I. A. Al-Yasir et.al., Trisection Open-Loop Varacter-Based Tunable Filter for 5G Wireless Communications, Proceedings of the 1st International Multi-Disciplinary Conference Theme: Sustainable Development and Smart Planning, 28-30 June 2020, Cyberspace, pp. 315-322.

[45] Atta Ullah and et.al., Dual-Band MIMO Antenna System for Next Generation Smartphone Applications, Proceedings of the 1st International Multi-Disciplinary Conference Theme: Sustainable Development and Smart Planning, 28-30 June 2020, Cyberspace, pp. 331-338.

[46] Ilvonen J, et al. Mobile terminal antenna performance with the user's hand. IEEE Antenna and Wireless Propagation Letters. 2000, 10:772775. 